\title{
An Embedded Real-Time Biometric Recognition System for Defense system
}

\author{
${ }^{1}$ Muthuselvi M, PG Scholar, ${ }^{2}$ Mr.Manikandan S, Asst...Professor, \\ ${ }^{I}$ Department of Electronics and Communication Engineering, PSN College of Engineering and Technology, \\ Tirunelveli, Tamil Nadu, India, \\ ${ }^{2}$ Department of Electronics and Communication Engineering, PSN College of Engineering and Technology, \\ Tirunelveli, Tamil Nadu, India,
}

\begin{abstract}
In this project, we propose a real-time embedded finger-vein recognition system for authentication on mobile devices. The system is implemented on an embedded platform and equipped with a novel finger-vein recognition algorithm. The proposed system consists of three hardware modules: image acquisition module, embedded main board, and human machine communication module. The structure diagram of the system is 1 . The image acquisition module is used to collect finger-vein images. The Embedded main board including the Microcontroller chip, memory (flash), and communication port is used to execute the finger-vein recognition algorithm and communicate with the peripheral device. The human machine communication module (LED or keyboard) is used to display recognition results and receive inputs from users.
\end{abstract}

Index Terms: finger-vein recognition; biometrics; mobile Devices; DSP

\section{Introduction}

Private information is traditionally provided by using Passwords or Personal Identification Numbers (PINs), which are easy to implement but is vulnerable to the risk of exposure and being forgotten. Biometrics, which uses human physiological or behavioral features for personal Identification, has attracted more and more attention and is becoming one of the most popular and promising alternatives to the traditional password or PIN based authentication techniques [1]. Moreover, some multimedia content in consumer electronic appliances can be secured by biometrics [2]. There is a long list of available biometric patterns, and many such systems have been developed and implemented, including those for the face, iris, fingerprint, palm print, hand shape, voice, signature, and gait. Notwithstanding this great and increasing variety of biometrics patterns, no biometric has yet been developed that is perfectly reliable or secure. For example, fingerprints and palm prints are usually frayed; voice, signatures, hand shapes and iris images are easily forged; face recognition can be made difficult by occlusions or face-lifts [3]; and biometrics, such as fingerprints and iris and face recognition, are susceptible to spoofing attacks, that is, the biometric identifiers can be copied and used to create artifacts that can deceive many currently available biometric devices. The great challenge to biometrics is thus to improve recognition performance in terms of both accuracy and efficiency and be maximally resistant to deceptive practices. To this end, many researchers have sought to improve reliability and frustrate spoolers by developing biometrics that are highly individuating; yet at the same time, present a highly complex, hopefully insuperable challenge to those who wish to defeat them [4]. Specially for consumer electronics applications, biometrics authentication systems need to be cost-efficient and easy to implement [5].

The finger-vein is a promising biometric pattern for personal identification in terms of its security and convenience [6]. Compared with other biometric traits, the finger-vein has the following advantages [7]: (1) the vein is hidden inside the body and is mostly invisible to human eyes, so it is difficult to forge or steal. (2) The non-invasive and contactless capture of finger-veins ensures both convenience and hygiene for the user, and is thus more acceptable. (3) The finger-vein pattern can only be taken from a live body. Therefore, it is a natural and convincing proof that the subject whose finger-vein is successfully captured is alive. We designed a special device for acquiring high quality finger-vein images and propose a DSP based embedded platform to implement the finger-vein recognition system in the present study to achieve better recognition performance and reduce computational cost. The rest of this paper is organized as follows. An overview of the proposed system is given in Section 2. The device for finger-vein image acquisition is introduced in Section 3. Our recognition method is addressed in Section 4. Experimental results are then presented in Section 5. Finally, concluding remarks are given in Section 6. 


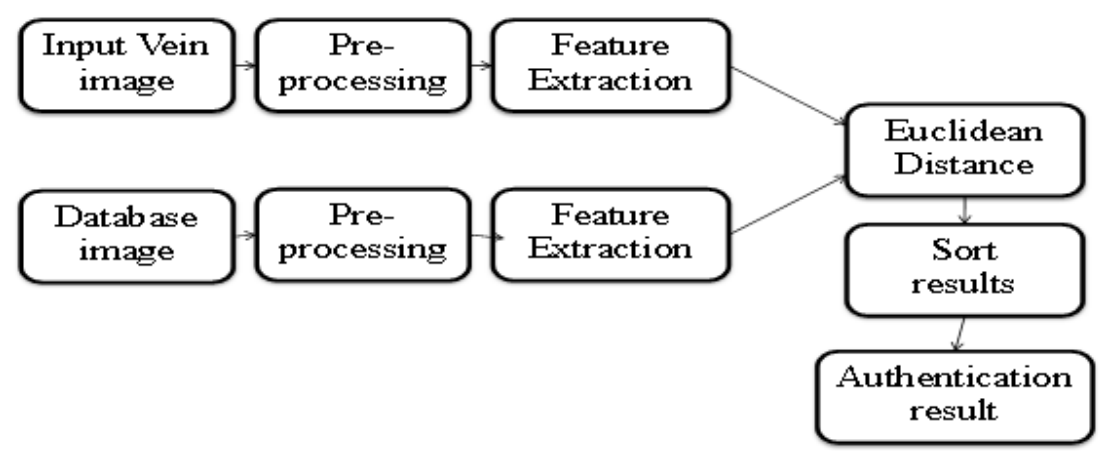

Fig.1.The flow-chart of the proposed recognition

\section{Overview Of The System}

The proposed system consists of three hardware modules: image acquisition module, DSP main board, and human machine communication module. The image acquisition module is used to collect finger-vein images. The DSP main board including the DSP chip, memory (flash), and communication port is used to execute the finger-vein recognition algorithm and communicate with the peripheral device. The human machine communication module (LED or keyboard) is used to display recognition results and receive inputs from users. (see fig.1.). The proposed finger-vein recognition algorithm contains two stages: the enrollment stage and the verification stage. Both stages start with finger-vein image pre-processing, which includes detection of the region of interest (ROI), image Segmentation, alignment, and enhancement. For the enrollment stage, after the pre-processing and the feature Extraction step, the finger-vein template database is built. For the verification stage, the input finger-vein image is extracted. Some different methods may have been proposed for finger-vein matching. Considering the computation complexity, efficiency, and practicability, however, we propose a novel method based on the fractal theory, which will be introduced in Section 4 in detail.

\section{IMAGE ACQUISITION}

To obtain high quality near-infrared (NIR) images, a special device was developed for acquiring the images of the finger vein without being affected by ambient temperature Generally, finger-vein patterns can be imaged based on the principles of light reflection or light transmission [8]. We developed a finger-vein imaging device based on light transmission for more distinct imaging. Our device mainly includes the following modules: a monochromatic camera of resolution $580 \times 600$ pixels, daylight cut-off filters (lights with the wavelength less than $800 \mathrm{~nm}$ are cut off), transparent acryl (thickness is $10 \mathrm{~mm}$ ), and the NIR light source. The structure of this device is illustrated in light-emitting diode (LED) was used as the illumination source for NIR light. With the LED illumination source, however, the shadow of the finger-vein obviously appears in the captured images. To address this problem, an NIR laser diode (LD) was used in our system Compared with LED, LD has stronger permeability and higher power. In our device, the wavelength of LD is $808 \mathrm{~nm}$.

\section{A. Image Segmentation and Alignment}

\section{Proposed Algorithm}

Because the position of fingers usually varies across different finger-vein images, it is necessary to normalize the images before feature extraction and matching. The bone in the finger joint is particular cartilage. Unlike other bones, it can be easily penetrated by NIR light. When a finger is irradiated by the uniform NIR light, the image of the joint is brighter than that of other parts. Therefore, in the horizontal projection of a fingervein image, the peaks of the projection curve correspond to the approximate position of the joints. Since the second joint of the finger is thicker than the first joint, the peak value at the second joint is less prominent. Hence, the position of the first joint is used for determining the position of the finger.

The alignment module includes the following steps. First, the part between the two joints in the fingervein image is segmented based on the peak values of the horizontal projection of the image. Second, a canny operator with locally adaptive threshold is used to get the single pixel edge of the finger. Third, the midpoints of finger edge are determined by edge tracing so that the midline can be obtained. Fourth, the image is rotated to adjust the midline of the finger horizontally. Finally, the ROI of the finger-vein image is segmented according to the midline.

\section{B. Image Enhancement}

The segmented finger-vein image is then enhanced to improve its contrast. The image is resized to $1 / 4$ of the original size, and enlarged back to its original size. Next, the image is resized to $1 / 3$ of the original size 
for recognition. Bucolic interpolation is used in this resizing procedure. Finally, histogram equalization is used for enhancing the gray level contrast of the image.

\section{Feature Extraction}

The fractal model developed by Mandelbrot [10] provides an excellent method for representing the ruggedness of natural surfaces and it has served as a successful image analysis tool for image compression and classification. Since different fractal sets with obviously different textures may share the same fractal dimension [11], the concept of lacunarity is used to discriminate among textures. The basic idea of lacunarity in many definitions is to quantify the "gaps or lacunae" presented in a given surface, which is used to quantify the denseness of a surface image. In this study, we focus on combining fractal and lacunarity measures for improving finger-vein recognition. When high gray level stands for white, the min operator of (1) will shrink the light regions corresponding to the particles, and the rate of this shrinking will only depend on the shape properties of the high gray level object. The max operator of (1), however, will shrink the background regions, and the rate of this shrinking will mainly be affected by the distribution of the high gray level object. In the case of finger-vein images, due to the directionality of the finger-vein, blanket growth can be made by directional maximizing (or minimizing) in the asymmetrical neighborhood instead of the symmetrical circular neighborhood. Considering the shape of the finger vein pattern, we modified (1) as follows, which can improve the rate of the shrinking and reveal the directional characteristics of the finger vein pattern.

\section{Box counting dimension (Minkowski dimension)}

Box counting is a method of gathering data for analyzing complex patterns by breaking a dataset, object, and Image. The image is divided into smaller and smaller pieces, typically "box"-shaped, and analyzing the pieces at each smaller scale. The box counting algorithms attempt to find an optimized way of cutting a pattern up that will reveal the scaling factor. The figure shows the extracted portion of the vein. In fractal geometry the Minkowski-Bouligand dimension, also known as Minkowski dimension or box-counting dimension is a way of determining the fractal dimension of a set. The box-counting dimension is one of a number of definitions for dimension that can be applied to fractals. For many well behaved fractals all these dimensions are equal; in particular, these dimensions coincide whenever the fractal satisfies the open set condition. Box-Counting of a D-dimensional array (with $\mathrm{D}=1,2,3$ ). $\quad[\mathrm{N}, \mathrm{R}]=\operatorname{BOXCOUNT}(\mathrm{C})$, where $\mathrm{C}$ is a Ddimensional array (with $\mathrm{D}=1,2,3$ ), counts the number $\mathrm{N}$ of $\mathrm{D}$-dimensional boxes of size $\mathrm{R}$ needed to cover the nonzero elements of $\mathrm{C}$. The box sizes are powers of two, i.e. $\mathrm{R}=1,2,4 \ldots 2^{\wedge} \mathrm{P}$, where $\mathrm{P}$ is the smallest integer such that MAX $(\operatorname{SIZE}(C))<=2^{\wedge} \mathrm{P}$. If the sizes of $\mathrm{C}$ over each dimension are smaller than $2^{\wedge} \mathrm{P}, \mathrm{C}$ is padded with zeros to size $2^{\wedge} \mathrm{P}$ over each dimension (e.g., a 320-by-200 image is padded to 512-by-512). The output vectors $\mathrm{N}$ and $\mathrm{R}$ are of size $\mathrm{P}+1$. For a RGB color image (m-by-n-by-3 array), a summation over the 3 RGB planes is done first.

The Box-counting method is useful to determine fractal properties of a 1D segment, a 2D image or a $3 \mathrm{D}$ array. If $\mathrm{C}$ is a fractal set, with fractal dimension $\mathrm{DF}<\mathrm{D}$, then $\mathrm{N}$ scales as $\mathrm{R}^{\wedge}$ (-DF). DF is known as the Minkowski-Bouligand dimension, or Kolmogorov capacity, or Kolmogorov dimension, or simply box-counting dimension. BOXCOUNT(C,'plot') also shows the $\log -\log$ plot of $\mathrm{N}$ as a function of $\mathrm{R}$ (if no output argument, this option is selected by default). BOXCOUNT(C,'slope') also shows the semi-log plot of the local slope DF = dlnN/dlnR as a function of R. If DF is constant in a certain range of R, then DF is the fractal dimension of the set $\mathrm{C}$. The derivative is computed as a 2 nd order finite difference (see GRADIENT). The execution time depends on the sizes of $\mathrm{C}$. It is fastest for powers of two over each dimension.

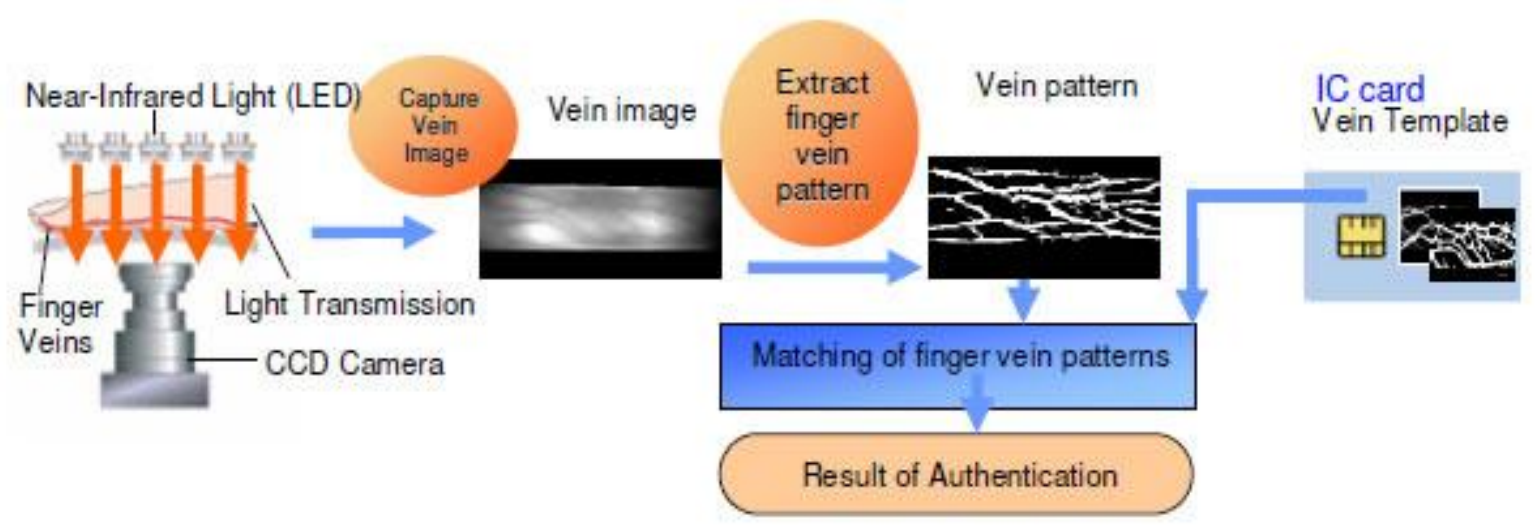

Fig. 2. Finger-vein recognition 


\section{A. Dataset}

\section{EXPERIMENTAL RESULTS}

To the best of our knowledge, is no public finger-vein image database has yet been introduced. Therefore, we constructed a finger-vein image database for evaluation, which contains finger-vein images from 100 subjects (55\% male and $45 \%$ female) from a variety of ethnic/racial ancestries. The ages of the subjects were between 21 years old and 58 years old. We collected finger-vein images from the forefinger, middle finger, and ring finger of both hands of each subject. Ten images were captured for each finger at different times (summer and winter). Therefore, there were a total of 6,000 finger-vein images in the database. Fig. 8 shows some example finger-vein images (after preprocessing) from different fingers.
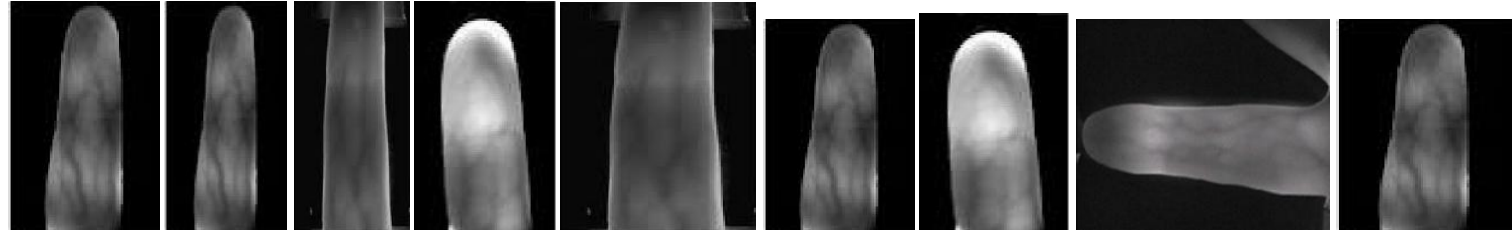

Fig. 3.Finger-vein images from different fingers

\section{B. Performance Evaluation}

The Vein geometry features are insufficient to identify individuals. This is because, the Vein geometry features such as Vein size, Vein width and others of adults are similar The Vein line features include principal lines, wrinkles and ridges. Ridges are the fine lines of the palm print. It requires high-resolution image or inked Vein image to obtain its features. The separation of wrinkles and principle lines are difficult since some wrinkles might be as thick as principle lines Vein point features use the minutiae points or delta points to identify an individual. Point features require high resolution hand image because low-resolution hand image does not have a clear point's location. Vein texture features are usually extracted using transform-based method such as Fourier Transform and Discrete Cosine Transform. Besides that, Wavelet transform is also used to extract the texture features of the Vein. In this work, a sequential modified Haar wavelet is proposed to find the Modified Haar Energy (MHE) feature Haar wavelet decomposes the vein image into approximation (A), horizontal detail $(\mathrm{H})$, vertical detail (V) and diagonal detail (D). The Modified Haar Energy (MHE) for each of the block is calculated using

$$
\operatorname{MHE}_{i, j, k}=\sum_{p=1}^{p} \sum_{q=1}^{Q}\left(\mathrm{C}_{p, q}\right)^{2}
$$

This method used to evaluate the performance of our proposed method.

\section{Comparison with Previous Methods}

Miura et al. [19] used a database that contained 678 different infrared images of fingers. These images were obtained from persons working in their laboratory aged 20 to 40, approximately $70 \%$ of whom were male. Song's [20] finger-vein image dataset contained 1,125 images collected using an infrared imaging device they built. Nine images were taken for each of 125 fingers. Compared with these databases, ours is larger and the data-collection interval is longer. Thus, our database is more challenging. Moreover, our system is implemented on a general DSP chip.

\section{CONCLUSION}

The present study proposed an end-to-end finger-vein recognition system based on the Minkowski dimension implemented on a DSP platform. The proposed system includes a device for capturing finger-vein images, a method for ROI segmentation, and a novel method combining blanket dimension features and lacunarity features for recognition. The images from 600 fingers in the dataset were taken over long time interval (i.e., from summer to winter) by a prototype device we built. Our system is suitable for application in mobile devices because of its relatively low computational complexity and low power consumption. 


\section{Reference}

[1] A. K. Jain, S. Pankanti, S. Prabhakar, H. Lin, and A. Ross, "Biometrics: a grand challenge", Proceedings of the 17th International Conference on Pattern Recognition (ICPR), vol. 2, pp. 935-942, 2004.

[2] P. Corcoran and A. Cucos, "Techniques for securing multimedia content in consumer electronic appliances using biometric signatures," IEEE Transactions on Consumer Electronics, vol 51, no. 2, pp. 545-551, May 2005.

[3] Y. Kim, J. Yoo, and K. Choi, "A motion and similarity-based fake detection method for biometric face recognition systems," IEEE Transactions on Consumer Electronics, vol.57, no.2, pp.756-762, May 2011.

[4] D. Wang , J. Li, and G. Memik, "User identification based on fingervein patterns for consumer electronics devices", IEEE Transactions on Consumer Electronics, vol. 56, no. 2, pp. 799-804, 2010.

[5] H. Lee, S. Lee, T. Kim, and Hyokyung Bahn, "Secure user identification for consumer electronics devices," IEEE Transactions on Consumer Electronics, vol.54, no.4, pp.1798-1802, Nov. 2008.

[6] D. Mulyono and S. J. Horng, "A study of finger vein biometric for personal identification", Proceedings of the International Symposium Biometrics and Security Technologies, pp. 134-141, 2008.

[7] Z. Liu, Y. Yin, H. Wang, S. Song, and Q. Li ,"Finger vein recognition with manifold learning", Journal of Network and Computer Applications, vol.33, no.3, pp. 275-282, 2010.

[8] Y. G. Dai and B. N. Huang, "A method for capturing the finger-vein image using non uniform intensity infrared light", Image and Signal Processing, vol.4, pp.27-30, 2008.

[9] X. Sun, C. Lin, M. Li, H. Lin, and Q. Chen, "A DSP-based finger vein authentication system", Proceedings of the Fourth International Conference on Intelligent Computation Technology and Automation, pp.333-336, 2011.

[10] B. B. Mandelbrot, Fractals: Form, Chance and Dimension, San Francisco, CA: Freeman, 1977.

[11] B. B. Mandelbrot and D. Stauffer, "Antipodal correlations and the texture (fractal lacunarity) in critical percolation clusters", Journal of Physics A: Mathematical and General, vol.27, pp.237-242, 1994.

[12] J. Berke, "Using Spectral Fractal Dimension in Image Classification", Innovations and advances in computer sciences and engineering, pp. 237-241, 2010.

[13] Z. Feng, "Variation and Minkowski dimension of fractal interpolation surface", Journal of Mathematical Analysis and Applications, vol. 345, no.1, pp. 322-334, 2008.

[14] S. Peleg and J. Naor, "Multiple resolution texture analysis and classification", IEEE Transactions on Pattern Analysis and Machine Intelligence, vol.6, no.4, pp.518-523, 1984.

[15] C. Allain and M. Cloitre, "Characterizing the lacunarity of random and deterministic fractal sets", Physical Review A, vol.44, no.6, pp. 3552- 3558, 1991.

[16] K. I. Kilic and R. H. Abiyev, "Exploiting the synergy between fractal dimension and lacunarity for improved texture recognition", Signal Processing, vol. 91, no. 10, pp. 2332-2344, 2011.

[17] Novianto, Suzuki, and Maeda, "Optimum estimation of local fractal dimension based on the blanket method," Transactions of the Information Processing Society of Japan, vol. 43, no.3, pp. 825-828, 2002.

[18] D. D. Hwang and I. Verbauwhede, "Design of portable biometric authenticators - energy, performance, and security tradeoffs," IEEE Transactions on Consumer Electronics, vol. 50, no. 4, pp. 1222-1231, Nov.2004.

[19] N. Miura, A. Nagasaka, and T. Miyatake, "Feature extraction of fingervein patterns based on repeated line tracking and its application to personal identification", Machine Vision Application, vol. 15, no.4, pp.194-203, 2004.

[20] W. Song, T. Kim, H. C. Kim, J. H. Choi, H. Kong and S. Lee, "A finger-vein verification system using mean curvature", Pattern Recognition Letters, vol. 32, no.11, pp. 1541-1547, 2011. 